\title{
A importância do envolvimento parental no desenvolvimento de crianças e adolescentes com epilepsia
}

\section{The Importance of Parental Involvement in the Development of Children and \\ Adolescents with Epilepsy}

\author{
Tânia Baptista \& Filomena Ermida da Ponte \\ FF/CS/Universidade Católica de Braga
}

\begin{abstract}
Resumo
A epilepsia é um distúrbio crónico caracterizado pela presença de crises recorrentes, resultantes de descarga excessiva dos neurónios. Salienta-se a dialética entre epilepsia, família e a escola, partindo do estudo comparativo de cinco casos. Os resultados indicam que as crianças/adolescentes epiléticos denotam predisposição para problemas nas tarefas de aprendizagem e insucesso escolar; autonomia/ajuste satisfatório nas rotinas diárias; limitações nas relações sociais. O envolvimento parental assume total importância. Existe antagonismo: os pais revelam preocupação e procuram assegurar a toma da medicação; mas, desvalorizam a prevenção da ocorrência das crises. A Escola responsabiliza-se por estruturar, implementar e avaliar as respostas educativas.

Palavras-chave: epilepsia, envolvimento parental, escola, desenvolvimento psicossocial.
\end{abstract}

\begin{abstract}
Epilepsy is a chronic disorder characterized by recurrent crises resulting from excessive discharge of neurons. Focus is on the dialectic between epilepsy, family and school, based on the comparative study of five cases. The results allow us to conclude that epileptics children/adolescents have predisposition to problems with the tasks of learning and school failure; satisfactory autonomy/adjustment in daily routines; limitations in social relations. It is crucial parental involvement. There is an antagonism: parents show concern and seek to ensure taking medication; but devalue the prevention of the occurrence of seizures. School assumes responsibility to organize, implement and evaluate educational responses.
\end{abstract}

Keywords: epilepsy, parental involvement, school, psychosocial development.

\section{Método}

Dada a natureza e o âmbito desta investigação, num amplo e complexo contexto das Ciências da Educação, optamos por uma investigação assente numa metodologia qualitativa. Utilizamos, também, para tratamento dos dados do inquérito por entrevista aos Encarregados de Educação e subsequente preenchimento de formulário e de alguns itens do inquérito por entrevista aos Professores de Educação Especial, a técnica de análise de dados qualitativos, através da apresentação dos resultados sob a forma de representações gráficas, permitindo descrever, de forma sucinta e evidente um conjunto de dados (Howell, 2010).

\begin{abstract}
Amostra
Utilizámos uma amostra não probabilística com amostragem por conveniência (Coutinho, 2011), constituída por cinco crianças com Epilepsia, de acordo com a Tabela 1 .
\end{abstract}

Tabela 1

Identificação da Amostra

\begin{tabular}{lccc}
\hline & Sexo & Idade & $\begin{array}{c}\text { Ano de } \\
\text { Escolaridade }\end{array}$ \\
\hline Criança A & Masculino & 11 & $5^{\circ}$ \\
Criança B & Feminino & 11 & $5^{\circ}$ \\
Criança C & Masculino & 12 & $6^{\circ}$ \\
Adolescente D & Feminino & 17 & $9^{\circ}$ \\
Adolescente E & Feminino & 16 & $10^{\circ}$ \\
\hline
\end{tabular}

Participam, também, os professores de Educação Especial das crianças que constituem a amostra $(\mathrm{N}=3)$, sendo dois do sexo feminino e um do sexo masculino, com uma longa experiência profissional no acompanhamento de discentes e respetivas famílias com patologias diversas.

\section{Instrumentos}

Utilizámos como instrumento uma Entrevista Baseada nas Rotinas (EBR). Trata-se de uma entrevista semiestruturada dirigida a pelo menos um dos pais ou familiares cuidadores, em relação ao funcionamento da criança e da família em rotinas diárias em casa e na escola. A EBR - A Entrevista Baseada nas Rotinas (adaptada de McWilliam, 2003) obedece a uma estrutura sequencial de onze itens, que constituem rotinas: (1) Hora de Dormir; (2) Acordar; (3) Vestir; (4) Hora do Banho; (5) Alimentação/Refeições; (6) Toma da Medicação; (7) Estudo/Tarefas Escolares; (8) Lazer/Ver Televisão/ Computador; (9) Família/ Escola/ Comunidade; (10) Interesses extracurriculares; (11) Prevenção/Ocorrência de Crises Epiléticas. Cada um dos itens pressupõe uma análise em três dimensões funcionais: (i) envolvimento; (ii) autonomia; (iii) relações sociais da criança e adolescente com epilepsia. 
De forma a poder documentar as informações recolhidas da EBR, procedemos à elaboração do respetivo Formulário Combinado EBR-EASFR, com adaptações ao estudo em causa. Consiste num formulário estruturado no qual as rotinas domésticas e as perguntas concretas se encontram especificadas e que resulta da combinação do formulário original da EBR e da Escala para a Avaliação da Satisfação das Famílias nas Rotinas adaptado de Scott e McWilliam (2000).

Por último, foi realizada uma entrevista aos Professores de Educação Especial, constituída por 13 questões abertas, de modo a poder aplicar-se de forma pouco rígida e aberta, tendo a especial atenção em evitar questões demasiado circunscritas ou inibitórias. O objetivo da entrevista foi abrir a área livre dos dois interlocutores no que respeita à matéria da entrevista, de forma a reduzir a área secreta do entrevistado e a área cega do entrevistador." (Carmo \& Fernades, 2008 ).

\section{Análise e discussão dos resultados}

Para melhor inteligibilidade e cruzamento relacional de variáveis recolhidas em dois instrumentos distintos, obtidos por participantes também diferentes, serão apresentados em dois momentos: (i) análise do Formulário Combinado EBR-EASFR, preenchido a partir das respostas recolhidas nas entrevistas aos Encarregados de Educação; (ii) análise de conteúdo das entrevistas realizadas aos Professores de Educação Especial.

\section{(i) Análise do Formulário Combinado EBR-EASFR adaptado de de Scott e McWilliam (2000).}

Na Figura 1, é possível verificar os resultados obtidos das respostas dadas pelos Encarregados de Educação no que concerne ao grau de envolvimento parental para cada uma das rotinas, de acordo com uma escala de três níveis: Podre, Médio e Excelente.

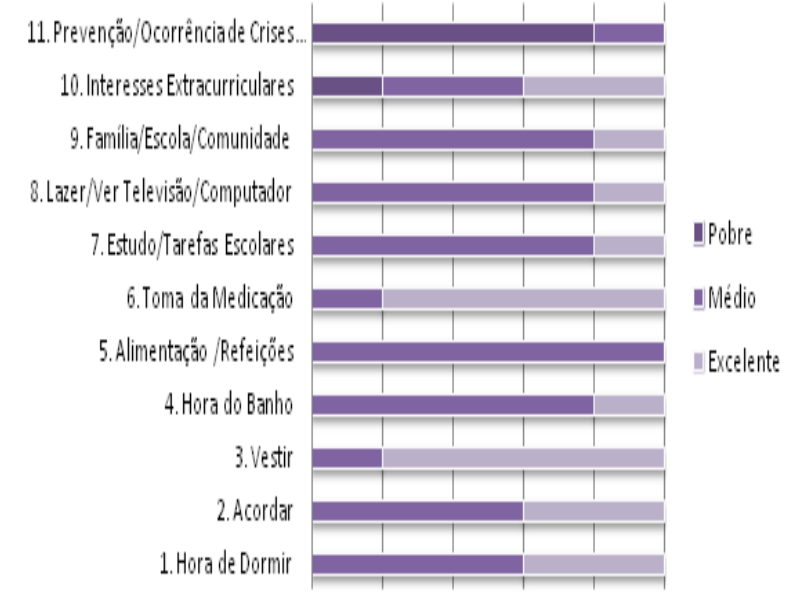

Figural - Grau de Envolvimento Parental nas Rotinas de Crianças e Adolescentes com Epilepsia

Assim, as rotinas em que se verifica um maior envolvimento das famílias são a "3. Vestir" e "6. Toma de Medicação", que reúnem $80 \%$ das respostas classificadas como "Excelente". Num extremo oposto, podemos concluir que a rotina que denota um pior envolvimento dos pais é a "11. Prevenção/Ocorrência das Crises Epitéticas”, em que $80 \%$ das informações obtidas apontam para um envolvimento "Pobre". A rotina "10. Interesses Extracurriculares" é a que reúne respostas mais díspares, sendo que $40 \%$ das informações sugerem um grau de envolvimento "Excelente" ou "Médio" e 20\% "Pobre". As restantes rotinas, na sua generalidade, apresentam um grau de envolvimento médio por uma parte significativa dos pais e respetivas famílias.

Relativamente ao grau de autonomia, os dados recolhidos e apresentados na Figura 2, permitem-nos concluir que são positivos em grande parte das rotinas, com exceção do estudo e tarefas escolares e prevenção/ocorrência de crises epiléticas.

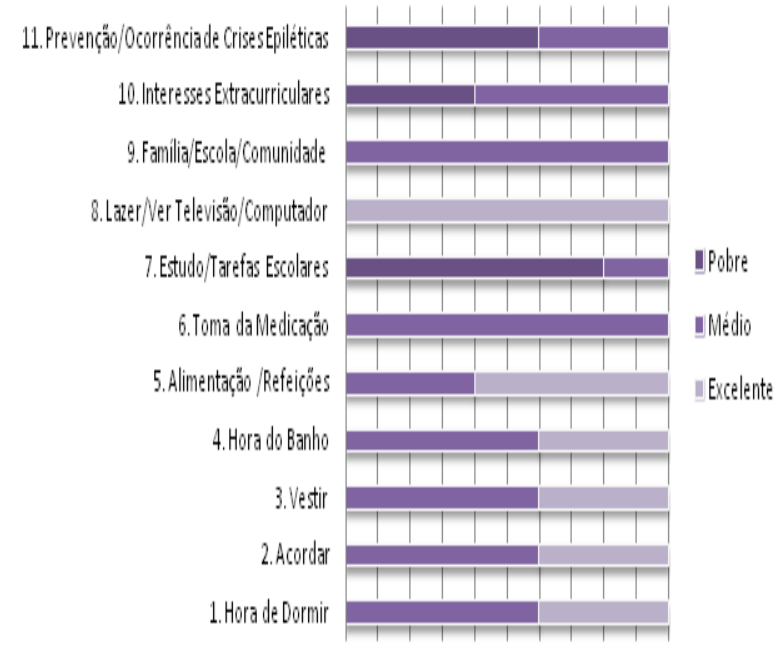

Figura 2 - Grau de Autonomia das Crianças e Adolescentes com Epilepsia nas Rotinas (\%)

Todas as crianças e adolescentes (100\%) revelaram autonomia "Excelente" na rotina "8. Lazer/Ver Televisão/Computador", atividades encaradas com grande agrado e às quais dedicam aproximadamente uma a duas horas diárias, distribuídas pela observação de programas televisivos e pelo uso do computador para aceder à Internet ou jogos. O momento da rotina “5.Alimentação/refeições", também parece reunir agrado por parte da amostra, sendo que $60 \%$ demonstra uma autonomia "Excelente". Nesta rotina tentámos aferir se as crianças e adolescentes com epilepsia necessitavam de ajuda para comer e se contribuíam para a preparação da refeição. A maior parte das crianças aprecia este momento em família e participa em tarefas como pôr e levantar a mesa, mas não na confeção das refeições. A rotina em que a amostra demonstra uma menor autonomia é a "7. Estudo/Tarefas Escolares", em que $80 \%$ dos casos foi classificada como "Pobre" e, apenas $20 \%$ como "Média". Na verdade nenhuma das crianças e adolescentes consegue realizar os trabalhos de casa e organizar o estudo sem qualquer tipo de ajuda. Em 80\% dos casos, apesar da ajuda fornecida pelos pais e familiares na execução das tarefas escolares, os resultados alcançados são insatisfatórios dando origem a alguma tensão e ansiedade no seio familiar. O fraco 
rendimento escolar é uma das preocupações principais partilhada por pais e professores. Em relação ao verificado no gráfico anterior, $60 \%$ das crianças e adolescentes em estudo demonstram uma autonomia "Pobre" no que diz respeito à rotina "11. Prevenção/Ocorrência de Crises Epiléticas", facto que parece estar associado à falta de envolvimento por parte das suas famílias no esclarecimento e valorização da mesma. Este estudo demonstrou que as crianças precisam de ser lembradas sobre o cumprimento de horários, nomeadamente no que diz respeito à hora de dormir, acordar, vestir e tomar banho. Apesar de não apresentarem recusa à realização das mesmas, necessitam de orientação por parte dos pais. Os constrangimentos ao nível da autonomia que, por vezes, são identificados nas crianças com epilepsia, resultam, sobretudo de uma reação às atitudes dos pais de medo, ansiedade, raiva, mágoa, culpa, e reações de superproteção, permissividade, super-indulgência além das dificuldades que sentem na imposição de limites disciplinares e estabelecimento de punições. Face a isto, a criança dá uma resposta que pode manifestar insegurança, imaturidade, dependência, falta de autonomia e baixa autoestima (Souza, Nista, Scotoni, \& Guerreiro, 1998).

Relativamente às competências sociais das crianças e adolescentes com epilepsia, os resultados patentes na Figura 3, sugerem um grau médio nas relações sociais, na generalidade das rotinas. Não obstante, quando comparada com as restantes variáveis em análise, parece ser a que denota pior desempenho, uma vez que na maioria das rotinas é frequente verificar a classificação "Pobre", ainda que pouco representativa, com um claro predomínio do "Médio" nas relações sociais e um menos representativo "Excelente", com exceção das rotinas "5.Alimentação/Refeições" e "3.Vestir".

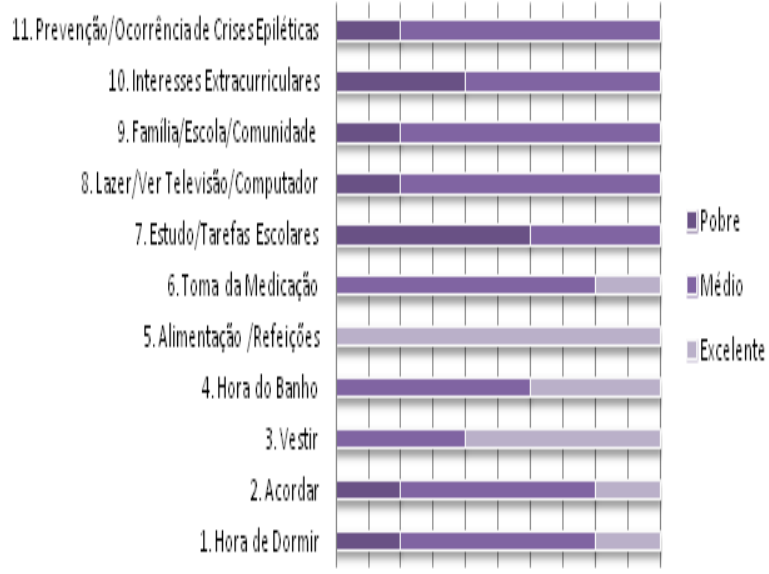

Figura 3 - Grau das Relações Sociais das Crianças e Adolescentes com Epilepsia

De facto, em consonância com o verificado no gráfico anterior, a rotina "7. Estudo/Tarefas Escolares" é a que evidencia um pior desempenho em termos de relações sociais, já que em $60 \%$ dos casos foi classificado como "Pobre" e nos restantes 40\% como "Médio". O fraco desempenho escolar das crianças e adolescentes com epilepsia afeta as relações sociais, uma vez que os pais revelaram falta de competências e impotência para lidar com esta problemática, em resultado das grandes dificuldades de aprendizagem, como ficou patente nos depoimentos prestados nas entrevistas. Este momento é, então, encarado com desagrado e resistência por parte das crianças e pais. Mais uma vez, a rotina "5. Alimentação/Refeições" parece ser aquela que apresenta um desempenho ótimo, sendo que $100 \%$ da amostra revela um "Excelente" grau nas relações sociais. Para este facto, parece contribuir a realização das refeições em família, altura de convívio e de partilha das experiências e assuntos vividos ao longo do dia.

(ii) análise de conteúdo das entrevistas realizadas aos Professores de Educação Especial.

Adotando a metodologia proposta por Vala (2009), dividimos as entrevistas em categorias, apresentando os contributos de cada professor de forma comparativa e simultânea, segundo a terminologia que se apresenta: PA - Professor A; PB - Professor B e PC - Professor C. A construção das categorias foi feita à posteriori, sendo que as referências teóricas serviram de orientação à primeira exploração do material, contribuindo para a reformulação e alargamento das hipóteses e da problemática em estudo. Esta etapa revelou-se talvez um dos momentos mais delicados desta investigação a par da escolha das unidades de análise e respetiva identificação. Foram estas as categorias que apuramos para explanar a importância do envolvimento dos agentes e do meio, em interação com as crianças e adolescentes com Epilepsia: Categoria A - Epilepsia; Categoria B - Família; Categoria C - Escola; Categoria D - Relação Escola-Família.

A Figura 4 permite-nos concluir que a referência às várias características comuns, não é aleatória, nem se distribui de forma homogénea ao longo dos três discursos. Embora cada um dos professores tenha enfatizado uma ou outra dificuldade, o que nos parece evidente é que as "Dificuldades de Aprendizagem" são as que aparecem com maior frequência (13) nos três discursos, seguindo-se das "Dificuldades de Interação com Pares" (7). Dos problemas em comum, apenas as "Dificuldades de Aprendizagem “ e "Défice de Atenção/Concentração" foram mencionados pelos três professores, enquanto que a "Lentidão na execução das Tarefas" foi mencionado por apenas um professor (B).

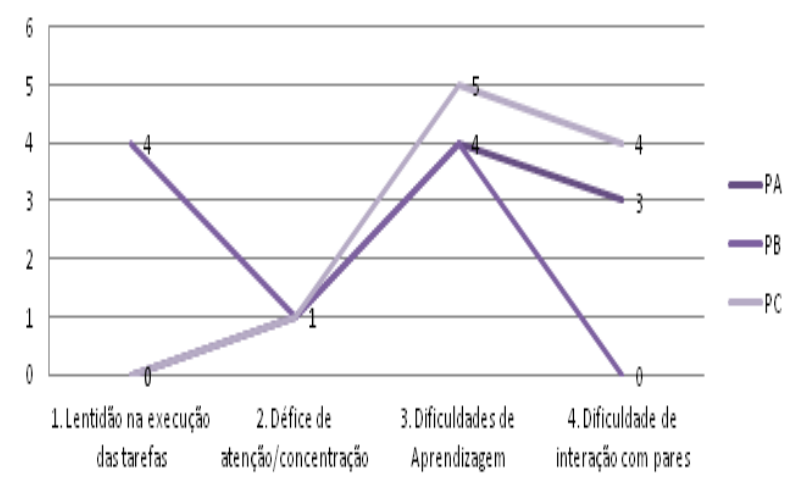

Figura 4 - Ocorrência das Características Comuns nas Crianças e Adolescentes com Epilepsia 
A epilepsia é uma doença que atinge muitas crianças e adolescentes mas, como não é facilmente identificável e pode assumir diversas manifestações, decorre de várias intensidades e frequências, dependendo de cada caso isoladamente, coloca obstáculos à real perceção do grande número de indivíduos afetados. As alterações que se verificam no desenvolvimento são decorrentes não só de fatores orgânicos, cognitivos/intelectuais, mas também pela forma como os outros se relacionam com o epilético.

(i) Procuramos, considerando as variáveis comummente associadas à patologia e possíveis repercussões, perceber a importância da envolvência parental no desenvolvimento de crianças e adolescentes com epilepsia.

As informações recolhidas não foram consensuais, embora os contributos de pais e professores tenham enfatizado limitações diferentes consoante os indivíduos em análise: lentidão na execução das tarefas; défice de atenção/concentração; dificuldades de aprendizagem; dificuldade de interação com pares. Estes resultados permitem-nos perceber uma certa predisposição para problemas associados às tarefas de aprendizagem e insucesso escolar. As crianças epiléticas constituem-se como um grupo educacional vulnerável, apresentando riscos para o baixo rendimento académico, além de ajustamento psicossocial pobre, o que pode resultar em absentismo e abandono da escola (Zanni, Matsukura \& Filho,2009).

Os dados recolhidos e analisados permitem-nos concluir que os pais não atribuem responsabilidades à escola pelo insucesso escolar e dificuldades de aprendizagem das crianças e adolescentes com epilepsia, apontando como principal fator responsável a própria patologia. As dificuldades evidenciadas pelos seus filhos e das limitações impostas pela própria epilepsia, também não impõem uma formulação objetiva dos aspetos de maior relevância e urgência. Este facto parece-nos estar associado a uma certa desresponsabilização pelo processo educativo, frequentemente atribuído à escola e aos agentes educativos.

Uma das preocupações que os pais frequentemente revelam sobre o aproveitamento escolar dos seus educandos relacionam-se com as expectativas e percursos escolares que perspetivam. O estudo confirmou a existência de fortes perspetivas de futuro que facilmente se desvanecem em resultado do insucesso escolar e das dificuldades de aprendizagem que vão sendo manifestadas. Estudos sugerem, ainda, uma relação de causalidade entre as expectativas pobres dos pais em relação à performance académica das crianças com epilepsia e o rendimento escolar, sendo que o mesmo fica seriamente comprometido e os resultados tendem a piorar (Long \& Moore 1979; Chugani et al., 1990).

O envolvimento parental reveste-se de uma total e inevitável importância, segundo a opinião dos professores de Educação Especial, este está patente em todas as rotinas diárias das crianças e adolescentes com epilepsia. É na família que a criança é gerada, protegida e se desenvolve ao longo da sua vida, pelo que concordamos com um conceito sistémico de família, no seio da qual a criança cresce e desenvolve a sua personalidade, autonomia e relações com o ambiente.

A primeira grande constatação suscitou alguma perplexidade, devido a certo antagonismo por parte das famílias na postura face à doença: se por um lado os pais revelam uma grande preocupação e procuram assegurar uma correta e atempada toma da medicação; por outro lado, desvalorizam a prevenção da ocorrência das crises epiléticas e utilização de procedimento adequados. A prevenção das crises é, sem dúvida um aspeto a valorizar na prática quotidiana das famílias com crianças e adolescentes com epilepsia. Um esclarecimento adequado por parte dos familiares pode ser crucial para que a criança se mantenha calma e saiba o que esperar em caso da ocorrência de uma crise (Liga Portuguesa Contra a Epilepsia, 2006; Kutscher, 2011).

A principal razão por nós encontrada foi a intenção em não alarmar, nem dar importância excessiva a um acontecimento raro, dado tratarem-se de epilepsias controladas, na sua generalidade. Simultaneamente, estas crianças e adolescentes com epilepsia raramente demonstram abertura para falar sobre o assunto.

(ii) Posteriormente, foi nossa intenção conhecer o nível de condicionamento no desenvolvimento psicossocial de crianças $e$ adolescentes com epilepsia, decorrente da sobreproteção por parte dos pais;

$\mathrm{Na}$ generalidade, as preocupações e receios dos pais da crianças e adolescentes com epilepsia são comuns aos demais, independentemente de terem um filho saudável ou não, ou da especificidade da sua doença. O que parece evidente é que os professores denotam uma "ampliação" desses mesmos receios, uma vez que estas crianças e adolescentes poderão necessitar de maior acompanhamento e ver limitada a sua autonomia, ao longo das suas vidas. Alguns dos principais receios apontados por pais e professores relacionam-se com as perspetivas futuras, nomeadamente no que concerne à situação profissional, em idade adulta, da pessoa com epilepsia. Estudos demonstram que pessoas com epilepsia apresentam até duas vezes mais probabilidade de desemprego ou de se submeterem a subemprego quando comparadas com pessoas sem epilepsia. Os fatores justificativos são: o tipo e a frequência das crises epiléticas, a idade de início e duração da epilepsia, e os efeitos adversos, sobretudo os cognitivos, das drogas; diminuição da autoestima e da expectativa de realização; estigma efetivo e a discriminação (Miziara, Miziara, \& Muñoz, 2011).

A ocorrência de crises epiléticas e consequente risco de acidente não constitui um problema que atormente e interfira diretamente com o quotidiano do portador da patologia e, consequentemente, das respetivas famílias. Os indivíduos da nossa amostra e o tipo de epilepsias e crises manifestadas, não inspiram grandes preocupações, por se encontrarem facilmente controlados com $o$ tratamento medicamentoso. Pais e professores, tentam equilibrar os riscos e as proibições. Os resultados vão ao encontro de autores que defendem que o doente com epilepsia não deve ser colocado numa "redoma almofadada", até porque tal solução não seria a mais 
segura porque ao ser demasiado restritiva, anula qualquer sensação de bem-estar, quando o que se pretende é tratar a criança e a família, sob risco de culminar num resultado emocional péssimo (Kutscher, 2011).

Verificam-se, no entanto, procedimentos que denotam sobreproteção por parte das famílias das crianças e adolescentes com epilepsia, sobretudo nas preocupações manifestadas aos profissionais de educação. Vários autores consideram que efeitos indesejados e a significativa conotação psicossocial da patologia, podem conduzir a comportamentos de sobreproteção por parte dos pais e restante família, originando um rendimento académico mais baixo, comprometimento nas atividades do quotidiano e na independência, além de dificuldades nas relações interpessoais dentro e fora do ambiente escolar, independentemente do nível cognitivo (Campfield; Breau \& Campfield, 2001; Sillanpaa \& Cross, 2009; Tsuchie et al., 2006).

(iii) Um dos objetivos primordiais deste estudo consistia em avaliar a colaboração entre os Pais e a Escola, na estruturação, implementação e avaliação das respostas educativas;

Neste ponto, parece-nos que não estamos perante numa verdadeira colaboração: os professores assumem a responsabilidade em estruturar, implementar e avaliar as respostas educativas e os pais são informados acerca dos resultados evidenciados

Os contactos com os Encarregados de Educação deveriam ser mais valorizados por ambas as partes, já que possibilitariam uma maior interação capaz de beneficiar a estruturação, implementação e avaliação das respostas educativas.

Estamos convictos de que este processo fica aquém das suas amplas possibilidades, uma vez que a participação ativa dos pais que compreendem e vivem intrinsecamente a problemática dos seus educandos poderia ser uma mais-valia na atuação e regulação das respostas da escola.

Correia e Serrano (2008) justificam esta tensão na relação pais-professores pelo facto de num dos lados se encontrar pais com as suas frustrações $\mathrm{e}$ responsabilidades, e do outro, um professor/educador com escassa formação específica na área e com a responsabilidade de melhorar os seus conhecimentos e práticas. $\mathrm{O}$ culminar desta tensão traduz-se num clima de antagonismo entre estes agentes educativos, com prejuízos para a criança.

(iv) Por último, quisemos averiguar a importância da relação Escola - Família no sucesso escolar e no desenvolvimento psicossocial das crianças e adolescentes com Epilepsia.

Considerando o contexto histórico e cultural no desenvolvimento da identidade de cada criança, a escola pode funcionar como agente propulsor da formação das crianças e adolescentes com epilepsia. Pais e professores foram unânimes ao considerarem a inclusão e integração como aspetos essenciais para a promoção do desenvolvimento destas crianças, só possível através de uma profícua relação Escola - Família.

A escola e os seus professores quando acolhem alunos com Epilepsia devem saber como agir em conformidade com a especificidade da própria doença, pelo que as informações obtidas junto da família se revelam cruciais. Não obstante, dadas as características intrínsecas de cada criança e a forte possibilidade de ocorrência de crises epitéticas em espaço escolar, as preocupações evidenciadas enquadram-se mais no foro académico e não tanto na manifestação e controlo da doença, nomeadamente aquando da manifestação de crises. Este facto, a início causou-nos uma certa estranheza que depois é passível de ser compreendida, por se tratarem de crianças e adolescentes com epilepsias controladas que, raramente, apresentam crises na escola. A maioria dos professores considera ter um conhecimento regular ou insuficiente sobre esta patologia (Zanini, Cruz \& Zavareze, 2011).

As respostas obtidas não poderiam ser mais reconfortantes no que concerne ao desenvolvimento psicossocial, nomeadamente quanto à reduzida rejeição/estigma social, quando comparamos com a história recente. Na generalidade, tanto Encarregados de Educação como professores são da opinião de que essa rejeição não assume repercussões na atualidade, embora possam existir algumas situações esporádicas e pontuais de discriminação, de fácil resolução. Apesar de ainda se verificar um certa incompreensão, resultante de um, ainda, existente desconhecimento da doença, a verdade é que, atualmente, as evidências do estigma são menores, tendo sido abandonada a abordagem associada ao transcendental e divino. Para finalizar, e apesar dos constrangimentos e dificuldades nestas parcerias entre famílias e escola, acreditamos que seja uma luta diária, ao levantar todos os dias e enfrentar as dificuldades vividas por estas crianças e adolescentes. Fica, porém, sempre a recompensa de saber que todos (Família $\rightarrow$ Escola $\rightarrow$ Sociedade) rumam na direção do mesmo ideal: proporcionar um desenvolvimento psicossocial que lhes permita a obtenção de autonomia e um nível de vida digno.

\section{Referências}

Campfield, C., Breau, L., \& Campfield, P. (2001). Impact of pediatric epilepsy on the family. Epilepsia, pp. 104-112.

Carmo, H., \& Fernandes, M. M. (2008). Metodologia da Investigação. Guia para a autoaprendizagem. Lisboa: Universidade Aberta.

Chugani, H. T., \& al., e. (1990). Infantil spasms: 1. PET identifies focal cortical. Ann Neurol, pp. 406-413.

Correia, L. d., \& Serrano, A. M. (2008). Envolvimento Parental na educação do aluno com necessidades educativas especiais. In L. d. Correia, Inclusão $e$ Necessidades Educativas Especiais: Um guia para educadores e professores. Porto: Porto Editora, pp. 155-164.

Coutinho, C. P. (2011). Metodologia de Investigação em Ciências Sociais e Humanas: Teoria e Prática. Coimbra: Almedina.

Howell, D. C. (2010). Statistical Methods for Psychoology. Belmont: Wadsword. 
Kutscher, M. L. (2011). Compreender a Epilepsia - Um guia para Pais, Professores e Outros Profissionais. Porto: Porto Editora.

Long, C. G., \& Moore, J. R. (1979). Parental expectations for their epileptic children. J. Child Psychol Psychiatric, pp. 299-312.

McWilliam, R. A. (2003). RBI report form. Nashville, TN: Vanderbilt University Medical Center.

Miziara, C. S., Miziara, I. D., \& Muñoz, D. R. (2011). Epilepsy and work: when the epilepsy should be considered incapacitating? Saúde, Ética \& Justiça, pp. 103-110.

Scott, S., \& McWilliam, R. A. (2000). Scale for assessment of family enjoyment within routines (SAFER). University of North Carolina at Chapel Hill: FPG Child Development Institute.

Sillampaa, M., \& Cross, J. H. (2009). The psychosocial impact of epilepsy in childhood. Epilepsy \& Behavior , pp. 5-10.

Souza, E., Nista, C., Scotoni, A. E., \& Guerreiro, M. M. (1998). Sentimentos e reações de pais de crianças epilépticas. Arq Neuro-Psiquiatr, pp. 39-44.

Tsuchie, S. Y. (2006). What about us? Siblings of children with epilepsy. Seizure, pp. 610-614.

Vala, J. (2009). A Análise de Conteúdo. In A. S. Silva, \& J. M. Pinto, Metodologia Das cIências Sociais. Edições Afrontamento, pp. 101-128.

Zanini, R., Cruz, R., \& Zavareze, T. (2011). A Perceção dos Professores do Ensino Fundamental sobre a Criança com Epilepsia na Escola em Santa Catarina. Jounal of Epilepsy and Clinical Neurophysiology, pp. 33-40.

Zanni, K. P., Matsukura, T. S., \& Filho, H. d. (2009). Investigando a frequência escolar de crianças com epilepsia. Revista "Educação Especial" , pp. 391-408.

http://epilepsia.lvengine.net/Imgs/crises-e-seguranca.pdf Crises Epilépticas e Segurança Autor: Liga Portuguesa Contra a Epilepsia (publicação sobre normas de segurança na prevenção e ocorrência de crises epiléticas) acedido em outubro de 2013. 